\title{
Efficacy of denosumab in two cases with multiple- system atrophy and osteoporosis
}

This article was published in the following Dove Press journal:

Therapeutics and Clinical Risk Management

\author{
Masashi Uehara \\ Yukio Nakamura \\ Jun Takahashi \\ Takako Suzuki \\ Hiroyuki Kato \\ Department of Orthopaedic Surgery, \\ Shinshu University School of \\ Medicine, Matsumoto, Japan
}

\begin{abstract}
Background: Multiple-system atrophy (MSA) is an $\alpha$-synucleinopathy with a very aggressive course and poor prognosis, which lacks efficient treatment. Thus, MSA represents a serious health and social problem. Progressive stridor and acute laryngeal obstruction likely occur in MSA; however, little is known about the bone metabolism or efficacy of bone absorption drugs, such as denosumab, in osteoporosis with MSA.
\end{abstract}

Case presentation and methods: Two male patients with osteoporosis in MSA presented to our institution (at 54 and 68 years of age). Denosumab was started after the diagnosis of osteoporosis in MSA, and the therapy was continued for 18-24 months.

Results: Lumbar and hip bone mineral density showed a 3.5\% and $0.6 \%$ increase at 24 months or a $10.3 \%$ and $3.2 \%$ increase at 18 months, respectively. Bone turnover markers were also improved in the two cases during follow-up. No fractures or other complications were recorded during the observation period.

Conclusion: This is the first study describing osteoporosis in two patients with MSA being treated by osteoporotic treatment. Based on our findings, it can be concluded that denosumab may be an effective therapy for osteoporosis in MSA.

Keywords: bone metabolism, denosumab, multiple-system atrophy, osteoporosis, bone mineral density

\section{Introduction}

Multiple-system atrophy (MSA) is histologically a disease in which insolubilized $\alpha$-synuclein is accumulated in nerve cells and oligodendroglia, and neuronal death is promoted. ${ }^{1}$ Symptoms in the early stages are prominent in cerebellar ataxia, parkinsonism, and autonomic dysfunction. ${ }^{2}$ It is characterized that these three major symptoms overlap when progressed, and findings such as atrophy of the brain stem and cerebellum and abnormalities of the striatum are observed in diagnostic imaging.

In spinocerebellar degeneration, a disease similar to MSA, the bone mineral density (BMD) was found to be significantly reduced, particularly in women, which increased the risk of fracture over 10 years, compared with those in the control group. ${ }^{3}$ Sato et al have also described that bisphosphonate (BP) plus vitamin D supplementation may correct hypovitaminosis $\mathrm{D}$, thereby improving bone resorption and frequent falls. However, to the best of our knowledge, there has been no report on the bone and calcium metabolism and fracture incidence in MSA patients during osteoporotic treatment to date.

In this report, we discuss about two cases of MSA with osteoporosis treated by denosumab for 18-24 months. Consequently, bone metabolism and BMD substantially improved, and no fracture or adverse effects occurred. These findings suggest that denosumab could be a therapeutic option to treat osteoporosis in MSA patients. 


\section{Case presentation}

\section{Patient I}

A 54-year-old man was taking talutireline hydrate for MSA that was diagnosed at the age of 51 years. The diagnosis of MSA by an experienced neurologist was based on trunk disorder, swallowing disorder, dysarthria, and MRI finding of cerebellar atrophy. He suffered from vertebral compression fracture because of a minor fall at the age of 54 years. His lumbar and total hip BMD values were 1.071 and $0.933 \mathrm{~g} / \mathrm{cm}^{2}$, respectively. We commenced denosumab and oral supplementation with vitamin D and calcium $(762.5 \mathrm{mg}$ of precipitated calcium carbonate, 200 IU of cholecalciferol, and $59.2 \mathrm{mg}$ of magnesium carbonate). No complications including hypocalcemia occurred during the treatment course (Table 1). His lumbar and hip BMD values improved to 1.109 (3.5\% increase) and 0.939 (0.6\% increase), respectively, at 24 months afterward, although lumbar BMD was decreased at 18 months of treatment (Table 2 and Figure 1). Bone alkaline phosphatase (BAP), urinary cross-linked N-terminal telopeptides of type I collagen (NTX), and serum tartrate-resistant acid phosphatase $5 \mathrm{~b}$ (TRACP-5b) were suppressed during the treatment (Table 3 and Figure 2). No fractures occurred over the 2 years of denosumab treatment. As his disease condition of MSA became worse around 12 months after the start of denosumab therapy, he started using wheel chair, whereas he could do his own things independently before then.

\section{Patient 2}

A 68-year-old man was taking talutireline hydrate for MSA that was diagnosed at the age of 65 years. The diagnosis of MSA by an experienced neurologist was based on trunk disorder, muscle weakness in body trunk and lower limbs, cooperative movement disorder, orthostatic hypotension, and MRI finding of olivopontocerebellar atrophy. He suffered from femoral neck fracture and performed humeral head placement at the age of 67 years. His lumbar and total hip BMD values were 0.728 and $0.685 \mathrm{~g} / \mathrm{cm}^{2}$, respectively. We commenced denosumab without vitamin $\mathrm{D}$ or calcium at 1 year after surgery as he refused to take it due to the difficulty in swallowing vitamin D or calcium. No complications including hypocalcemia occurred during the treatment course (Table 1). His lumbar and hip BMD values improved to 0.803 (10.3\% increase) and 0.707 (3.2\% increase), respectively, at 18 months afterward (Table 2 and Figure 1). No fractures occurred during denosumab treatment. As his disease condition of MSA became worse around 6-8 months after the start of denosumab therapy, he started using a T-shaped case, whereas he could walk without any items before then. 

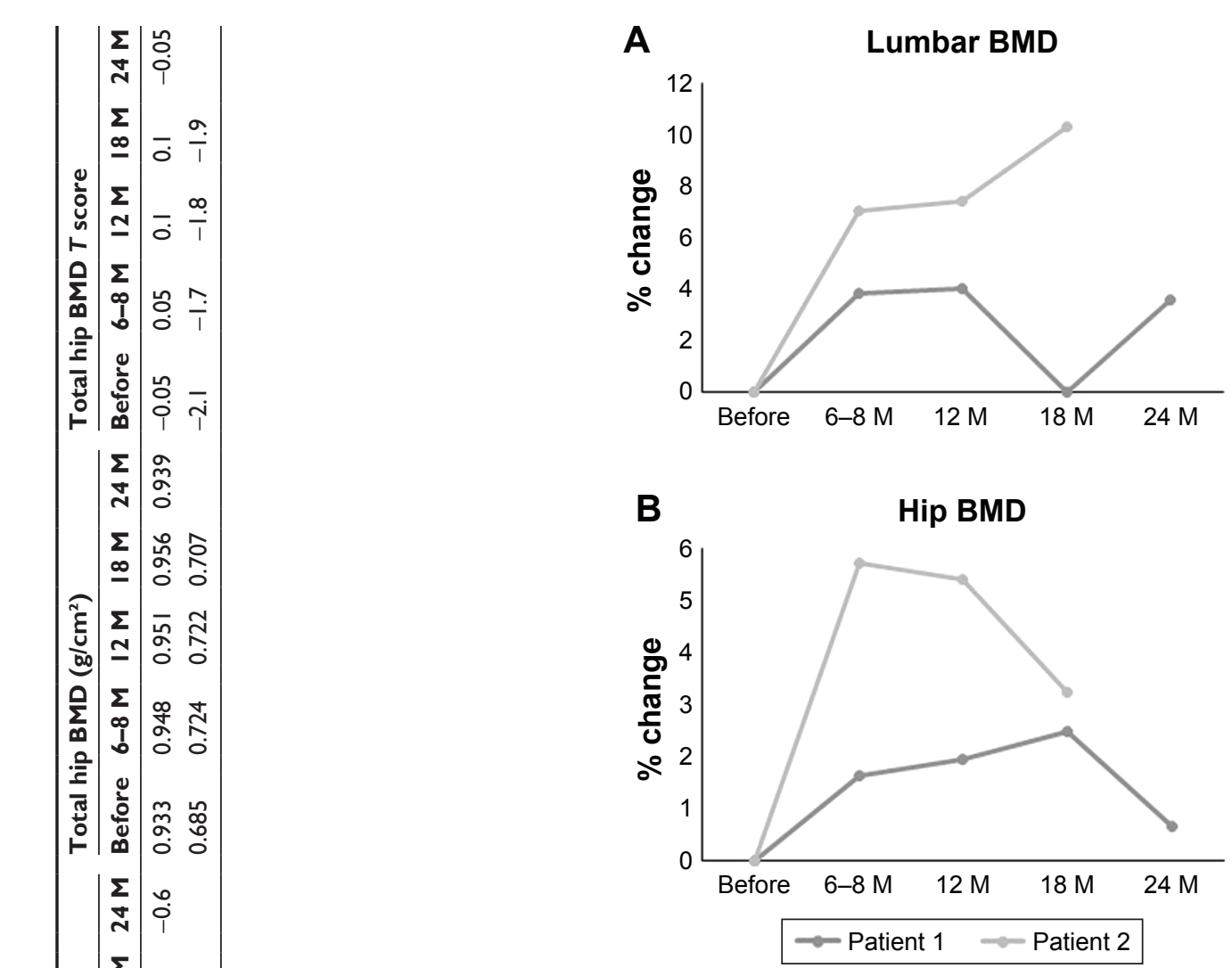

Figure I Percentage changes in lumbar BMD $(\mathbf{A})$ and total hip BMD $(\mathbf{B})$ before, and at $6-8,12,18$, and 24 months after denosumab therapy. Note: Patient 2 was followed up for 18 months, not 24 months. Abbreviations: BMD, bone mineral density; M, months.

BMD was measured using a dual-energy X-ray absorption fan-beam bone densitometer (Lunar Prodigy; GE Healthcare Bio-Sciences Corp., Piscataway, NJ, USA) at the L1-4 levels of the posteroanterior spine and bilateral total hips. BAP was measured as a bone formation marker by a chemiluminescent enzyme immunoassay, urinary NTX and TRACP-5b were measured by an enzyme-linked immunosorbent assay, and $1,25(\mathrm{OH})_{2} \mathrm{D}_{3}$ was measured by an immunoradiometric assay.

This study was approved by the institutional ethical review board at Shinshu University School of Medicine (\#2365) prior to its start and was in accordance with the ethical standards laid down in the 1964 Declaration of Helsinki for research involving human subjects. Written informed consent was provided by the two patients to have the case details and any accompanying data published.

\section{Discussion}

This report is the first of its kind on osteoporosis with MSA treated by denosumab therapy. BMD and bone turnover markers substantially improved at 18-24 months of denosumab therapy in the two cases in this study. Thus, denosumab 
might be a treatment choice for osteoporosis complicated with MSA.

To our knowledge, there has been no report on the bone metabolism, BMD, or fracture incidence in osteoporosis with MSA after osteoporosis treatment to date. However, disabling neurological diseases like spinocerebellar degeneration or multiple sclerosis, which are similar to MSA, are considered to have likely low BMD and high fracture incidence. ${ }^{3,4}$ Thus, osteoporosis and bone health should be considered in all patients with both inflammatory and degenerative chronic neurological diseases.

We have previously reported that denosumab increased BMD and improved bone turnover markers in primary as well as secondary osteoporosis, ${ }^{5,6}$ thereby preventing fractures. This study showed that denosumab therapy substantially enhanced BMD and improved bone turnover markers in two osteoporosis cases with MSA during the observational period, although the effect of denosumab might have been weak after 12-18 months of therapy, potentially because the activities of daily life (ADLs) of each patient had been decreased with the progression of MSA.

Although BPs are the first-line drugs according to the Japanese guidelines, ${ }^{7}$ due to their swallowing difficulties, we chose denosumab therapy this time as we have previously reported that denosumab was useful for secondary osteoporosis, such as anorexia nervosa and Duchenne muscle dystrophy. ${ }^{8,9}$

MSA is a rapidly progressive neurodegenerative disorder with poor prognosis and has no pharmacological or notable rehabilitation strategies available to prevent its worsening. Thus, it is required to improve the ADLs and quality of life of the patients with MSA. There has been little evidence of bone metabolism associated with MSA until the present cases. Thus, it is expected that our findings will serve as the basis of future research.

\section{Limitations}

The limitations of the current study include small sample size and retrospective design.

\section{Conclusion}

This study is the first to report on denosumab treatment for osteoporotic MSA patients in Japan. Denosumab produced substantial improvements in BMD and bone turnover markers with no fracture during the therapy, and therefore represents an effective treatment option for osteoporosis with MSA. 

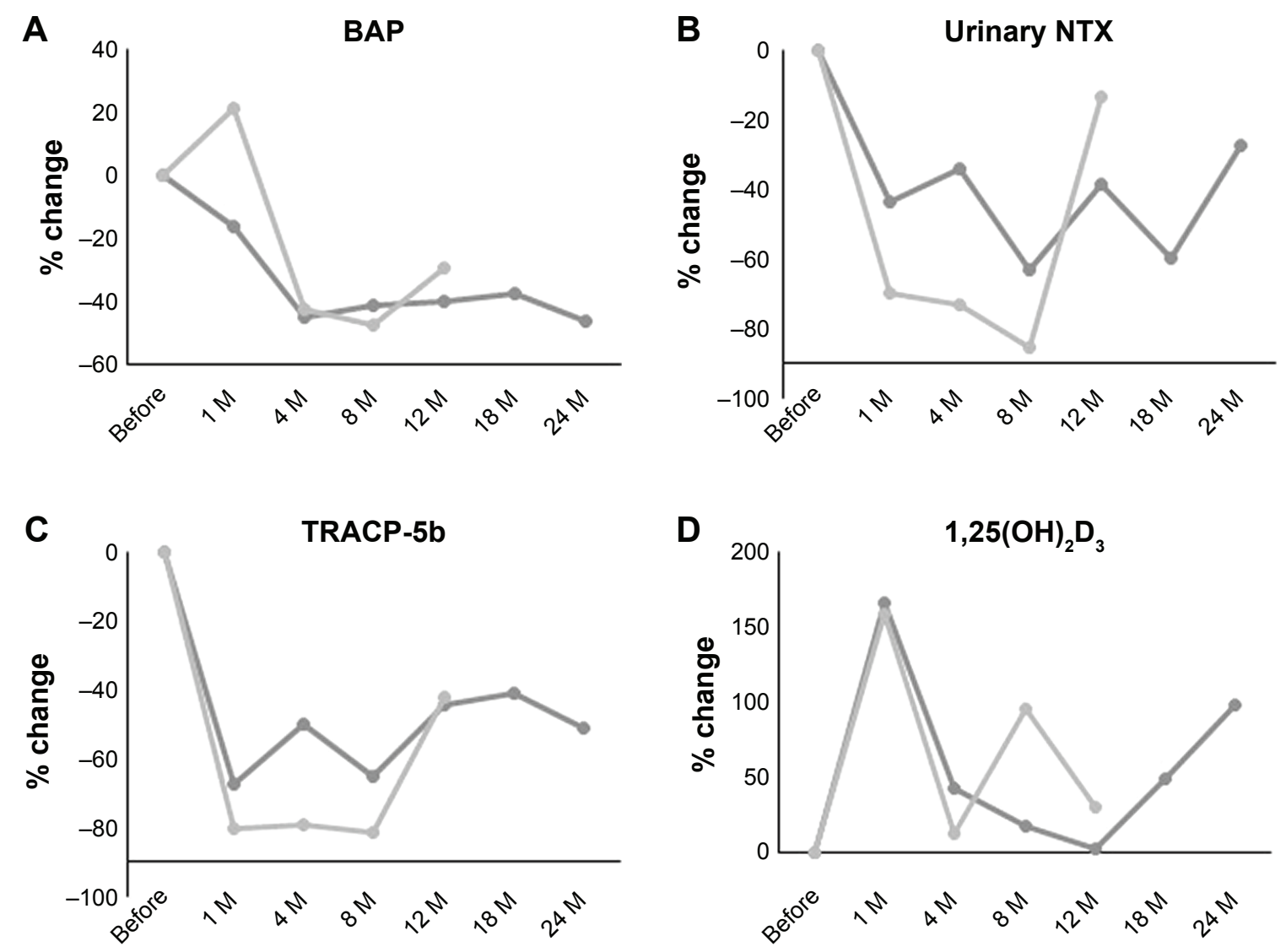

$\leadsto$ Patient $1 \multimap$ Patient 2

Figure 2 Percentage changes in BAP (A), urinary NTX (B), serum TRACP-5b (C), and active form of vitamin D or I, 25(OH) $\mathrm{D}_{3}(\mathbf{D})$ before, and at I, 4, 8, I2, I8, and 24 months after denosumab therapy.

Note: Patient 2 was followed up for 18 months, not 24 months.

Abbreviations: BAP, bone alkaline phosphatase; NTX, cross-linked N-terminal telopeptides of type I collagen; TRACP-5, tartrate-resistant acid phosphatase 5b; M, months.

\section{Author contributions}

YN directed this study. MU and TS collected patients' data. MU, JT, and YN wrote the main manuscript. HK gave suggestions on this study. All authors contributed toward data analysis, drafting and critically revising the paper, reviewed the manuscript, gave final approval of the version to be published, and agree to be accountable for all aspects of the work.

\section{Disclosure}

The authors report no conflicts of interest in this work.

\section{References}

1. Fanciulli A, Wenning GK. Multiple-system atrophy. $N$ Engl J Med. 2015;372(3):249-263.

2. Gilman S, Wenning GK, Low PA, et al. Second consensus statement on the diagnosis of multiple system atrophy. Neurology. 2008;71(9): 670-676.

3. Sato Y, Honda Y, Asoh T, Iwamoto J. Longitudinal study of bone and calcium metabolism and fracture in spinocerebellar degeneration. Eur Neurol. 2006;56(3):155-161.
4. Simonsen CS, Celius EG, Brunborg C, et al. Bone mineral density in patients with multiple sclerosis, hereditary ataxia or hereditary spastic paraplegia after at least 10 years of disease - a case control study. BMC Neurol. 2016;16(1):252.

5. Nakamura Y, Suzuki T, Kato H. Denosumab significantly improves bone mineral density with or without bisphosphonate pre-treatment in osteoporosis with rheumatoid arthritis: denosumab improves bone mineral density in osteoporosis with rheumatoid arthritis. Arch Osteoporos. 2017;12(1):80.

6. Nakamura Y, Suzuki T, Yoshida T, Yamazaki H, Kato H. Vitamin D and calcium are required during denosumab treatment in osteoporosis with rheumatoid arthritis. Nutrients. 2017;9(5):E428.

7. Orimo H, Nakamura T, Hosoi T, et al. Japanese 2011 guidelines for prevention and treatment of osteoporosis - executive summary. Arch Osteoporos. 2012;7:3-20.

8. Isobe F, Nakamura Y, Suzuki T, Kato H. Effects of denosumab on osteoporosis in three cases with anorexia nervosa and a review of the literature. Mod Rheumatol Case Rep. 2018;2(1).

9. Kumaki D, Nakamura Y, Sakai N, et al. Efficacy of denosumab for glucocorticoid-induced osteoporosis with Duchenne muscular dystrophy: a case report. JBJS Case Connect. Epub April 11, 2018. 


\section{Publish your work in this journal}

Therapeutics and Clinical Risk Management is an international, peerreviewed journal of clinical therapeutics and risk management, focusing on concise rapid reporting of clinical studies in all therapeutic areas, outcomes, safety, and programs for the effective, safe, and sustained use of medicines. This journal is indexed on PubMed Central, CAS,

EMBase, Scopus and the Elsevier Bibliographic databases. The manuscript management system is completely online and includes a very quick and fair peer-review system, which is all easy to use. Visit http://www.dovepress.com/testimonials.php to read real quotes from published authors.

Submit your manuscript here: http://www.dovepress.com/therapeutics-and-clinical-risk-management-journal 